Title:

Author(s):

Submitted to:
Determination of Fission Neutron Transmission

Through Waste Matrix Material Using Neutron Signal Correlation From Active Assay of ${ }^{239} \mathrm{Pu}^{\star}$

Charles Hollas

Gaetano Arnone

Glenn Brunson

Kenneth Coop

Institute of Nuclear Materials Management (INMM)

Annual Meeting

July 28 - August 1, 1996

Naples, Florida

AU6 26 BS

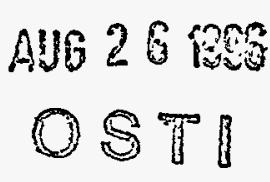

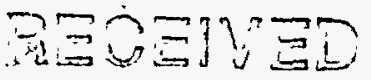
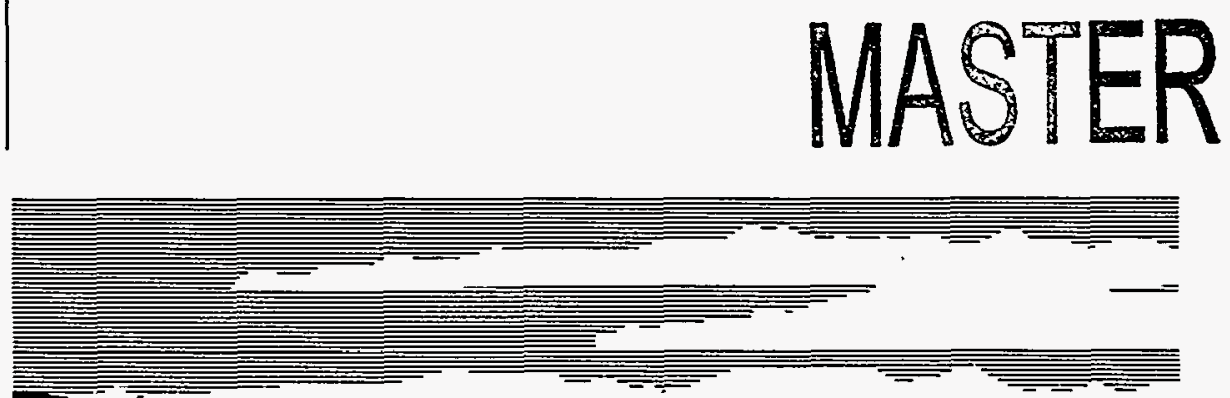

Los Alamos National Laboratory, an affirmative action/equal opportunity empldyer, is operated by the University of California for the U.S. Department of Energy under contract W-7405-ENG-36. By acceptance of this article, the publisher recognizes that the U.S. Government retains a nonexclusive, royalty-free license to publish or reproduce the published form of this contribution, or to allow others to do so, for U.S. Government purposes. The Los Alamos National Laboratory requests that the publisher identify this article as work performed under the auspices of the U.S. Department of Energy.

*This work supported by the U.S. Dept of Energy EM-50, Mixed Waste Foctßonther. 836 R5 


\section{DISCLAIMER}

Portions of this document may be illegible in electronic image products. Images are produced from the best available original document. 


\section{DISCLAIMER}

This report was prepared as an account of work sponsored by an agency of the United States Government. Neither the United States Government nor any agency thereof, nor any of their employees, makes any warranty, express or implied, or assumes any legal liability or responsibility for the accuracy, completeness, or usefulness of any information, apparatus, product, or process disclosed, or represents that its use would not infringe privately owned rights. Reference herein to any specific commercial product, process, or service by trade name, trademark, manufacturer, or otherwise does not necessarily constitute or imply its endorsement, recommendation, or favoring by the United States Government or any agency thereof. The views and opinions of authors expressed herein do not necessarily state or reflect those of the United States Government or any agency thereof. 


\title{
DETERMINATION OF FISSION NEUTRON TRANSMISSION THROUGH WASTE MATRIX MATERIAL USING NEUTRON SIGNAL CORRELATION FROM ACTIVE ASSAY OF ${ }^{239} \mathrm{PU}^{*}$
}

\author{
Charles L. Hollas, Gaetano Arnone, Glenn Brunson and Kenneth Coop \\ Advanced Nuclear Technology, NIS-6, Los Alamos National Laboratory \\ P.O. Box 1663, Mail Stop J562, Los Alamos, New Mexico 87545, USA \\ Telephone (505)-667-0677, Fax (505)-665-3657
}

\begin{abstract}
The accuracy of TRU (transuranic) waste assay using the differential die-away technique depends upon significant corrections to compensate for the effects of the matrix material in which the TRU waste is located. We have used a new instrument, the Combined Thermal/Epithermal Neutron (CTEN) instrument for the assay of TRU waste, to develop methods to improve the accuracy of these corrections. Neutrons from a pulsed 14-MeV neutron generator are moderated in the walls of the CTEN cavity and induce fission in the TRU material. The prompt neutrons from these fission events are detected in cadmium-wrapped ${ }^{3} \mathrm{He}$ neutron detectors. We report new methods of data acquisition and analysis to extract correlation in the neutron signals resulting from fission during active interrogation. We use the correlation information in conjunction with the total number of neutrons to determine the fraction of fission neutrons transmitted through the matrix material into the ${ }^{3} \mathrm{He}$ detectors. This determination allows us to cleanly separate the matrix effects into two processes: matrix modification upon the neutron interrogating flux and matrix modification upon the fraction of fission neutrons transmitted to the neutron detectors. This transmission information is also directly applied in a neutron multiplicity analysis in the passive assay of ${ }^{240} \mathrm{Pu}$.
\end{abstract}

\section{The CTEN Instrument}

A new instrument for the assay of ${ }^{239} \mathrm{Pu}$ and ${ }^{235} \mathrm{U}$ waste contained in 55 and 83 gallon drums has recently been constructed at the Los Alamos National Laboratory. This instrument designated CTEN, for Combined Thermal/Epithermal Neutron interrogation, actively and passively assays waste containers. The active interrogation usès a pulsed $14-\mathrm{MeV}$ neutron source, and neutrons from thermal neutron induced fission are detected by cadmium- wrapped ${ }^{3} \mathrm{He}$ neutron detectors. The signals from the neutron detectors are amplified and converted to logic pulses via leading edge discriminators. These logic pulses are then input to a custom electronic module, the Pulse Arrival Time Recording module (PATRM) (Ref. 1) which sequentially records the pulse arrival times of the neutron signals.

\section{Experimental Procedure}

The thirty nine cadmium wrapped or "shielded" ${ }^{3} \mathrm{He}$ neutron detectors of the CTEN instrument were used to detect neutrons from fission events. Signals from these detectors were configured into fifteen channels of logic signal input to fifteen channels of the PATRM. At the onset of the VETO pulse, the PATRM disables all data inputs, writes a code marker to its internal memory at the next memory location, stops the internal clock counter and resets this counter to zero. Upon lifting of the VETO, the clock counter and all data inputs are enabled. The result of the measurement is a history of the signal arrival times relative to the end of the VETO pulse. The clock frequency was 5 megahertz allowing the signal arrival time to be measured with an accuracy of 0.2 microseconds. Up to a million events can be recorded before transferring the data to a small computer for analysis and archiving. The VETO input to the PATRM was the logic pulse of length 500 microseconds derived from the trigger pulse from the neutron generator. Thus the PATRM was disabled during the neutron pulse and for most of the time in which the "shielded" ${ }^{3} \mathrm{He}$ detectors were able to detect neutrons from the neutron generator. The majority of pulses recorded by the PATRM were from fission events produced by the interrogating thermal neutron flux. The neutron

"This work supported by the U.S. Dept. of Energy, EM-50, Mixed Waste Focus Area. 
generator provided neutron pulses at a 100 hertz rate and duration of about twenty microseconds.

The fissionable material samples consisted of a dispersed 7 gram sample of ${ }^{239} \mathrm{Pu}$, a 9 gram enriched ${ }^{235} \mathrm{U}$ foil, and a $620 \mathrm{mg}$ metallic foil of ${ }^{239} \mathrm{Pu}$. Six separate data sets for the $7 \mathrm{gm}$ plutonium oxide and 9 grams ${ }^{235} \mathrm{U}$ samples were recorded following 6000 pulses each from the neutron generator. Measurement was also made with the CTEN chamber empty to record background effects.

\section{Feynman Variance Analysis}

We have applied the reduced variance method of analysis developed initially by Feynman, deHoffmann and Serber (Ref. 2) and used by other investigators (Refs. 3,4 ) of spontaneous fissioning systems. The time history of the neutron events recorded in the PATRM module, are examined to construct the Feynman Variance parameters FEY $Y_{\text {MEAN }}$ and FEY CORR from Feynman or "random" triggered distributions. The Feynman distribution FN $_{\text {ORDER }}$, is formed by randomly opening an inspection time interval of fixed width, and then counting the number of neutron signals that are present within this time interval. A histogram array is then incremented according to the number of neutrons found, and the process is repeated for the duration of the measurement. The resulting distribution then contains the number of times that zero, one, two, three, etc. neutrons were found within the inspection interval. The FN ORDER distributions are converted to probability distributions $P_{\text {ORDER }}$ by dividing the FN ORDER $_{\text {by }}$ the number of times the inspection interval (NUM_INT) was opened.

$\mathrm{P}_{\mathrm{ORDER}}=\mathrm{FN}_{\mathrm{ORDER}} /($ NUM_INT $)$.

The first and second moments of the PORDER distributions are then formed.

$\operatorname{Mom} 1=\Sigma\left\{m * \mathrm{P}_{\mathrm{m}}\right\}$

$\operatorname{Mom} 2=\Sigma\left\{m *(m-1) * P_{m}\right\}$

The first moment is the average, or mean, number of neutrons found in the time interval .

$\mathrm{FEY}_{\mathrm{MEAN}}=\mathrm{Mom1}$
The first moment divided by the interval value is the average neutron counting rate during the measurement. The first moment multiplied by the number of intervals is the total number of neutrons detected during the measurement,

$\mathrm{N} 1=\mathrm{FEY}_{\text {MEAN }} *($ NUM_INT).

The second moment represents the variance of the distribution. The difference between the variance and the square of the mean is a measure of the amount of correlation present in the data.

$\mathrm{FEY}_{\mathrm{COR}}=\sum \mathrm{m}(\mathrm{m}-1) * \mathrm{P}_{\mathrm{m}}-\left(\sum \mathrm{m} * \mathrm{P}_{\mathrm{m}}\right) *\left(\sum \mathrm{m} * \mathrm{P}_{\mathrm{m}}\right)$

For a neutron source that has no correlation's, such as neutrons from an Americium-Lithium source, the above distributions are those of a pure Poisson distribution, and this difference is identically zero.

This difference multiplied by the number of intervals is then the number of correlated neutrons $\mathrm{N} 2$ present in the data,

\section{$\mathrm{N} 2=\mathrm{FEY} \mathrm{CORR} *\left(\mathrm{NUM} \_\mathrm{INT}\right)$.}

For a passive interrogation of a system containing a spontaneously fissioning system such as ${ }^{240} \mathrm{Pu}$ or ${ }^{252} \mathrm{Cf}$, the fission rate is constant with time, although the time between any two fission events is completely random. For a system such as ${ }^{235} \mathrm{U}$ or ${ }^{239} \mathrm{Pu}$, undergoing active interrogation in the CTEN instrument, the fission rate is not constant, but follows the exponential die-away time dependence of the thermal neutrons which are producing the fission events. In order to at least partially compensate for this time dependence due to the thermal neutron decrease, we form many separate Feynman distributions. The first distribution is for an interval starting 500 microseconds after the neutron generator burst. The inspection time interval was chosen to be 100 microseconds. The second begins one time interval later, the third two time intervals later, etc. For the data described here, the neutron generator produced a neutron burst every ten milliseconds. Thus 95 Feynman distributions were generated. From these Feynman distributions 95 values for N1 and N2 were calculated. Each of these distributions are for inspection intervals that are random in time with respect to any fission event. 


\section{Experimental Results}

\section{A. The time dependence of $\mathrm{N} 1$ and $\mathrm{N} 2$.}

The N1 and N2 distributions for the 7 gram sample, shown on a log scale in Fig. 1, both exhibit an exponential decrease over the region of $\sim 800$ to 3000 microseconds, with a time constant that is representative of the thermal neutron die-away time in the CTEN chamber. A sharper exponential decrease is observed at the earliest times measured, of 500 microseconds to the start of the second exponential near 800 microseconds in the $\mathrm{N} 1$ distribution. This early region still contains neutrons from the neutron generator burst that have not yet been completely cleared from the ${ }^{3} \mathrm{He}$ detector packages. The solid lines represent the results of least square fits to the

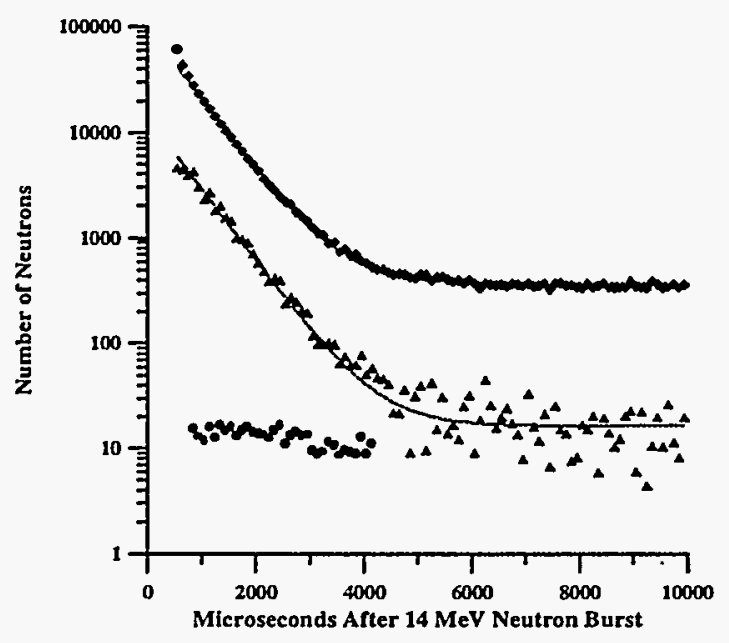

Fig 1. The time distributions for the N1 (diamonds), $\mathrm{N} 2$ (triangles) and ratio of $100 * \mathrm{~N} 2 / \mathrm{N} 1$ (circles) for the $7 \mathrm{gm}$ plutonium sample obtained with 6000 neutron generator pulses .

data for times greater than 800 microseconds, with the function

$$
Y(t)=A * \exp (-0.693 * t / \Psi)+C .
$$

Here $\Psi$ is the thermal neutron die-away half-life time constant in the empty CTEN chamber. The same value for $\Psi, 412.8$ microseconds, is used to generate the solid curves for both $\mathrm{N} 1$ and $\mathrm{N} 2$. The values for the ratio of N2/ N1 multiplied by 100 , are displayed as solid circles between 800 and 4000 microseconds after the neutron generator burst.

\section{B. Comparison to an Expanded Hage-Cifarelli Model.}

According to the results of Hage and Cifarelli (Ref. 3) for the passive analysis of spontaneous fission of ${ }^{240} \mathrm{Pu}$, the singles and doubles counting rates are related to the mass, detection efficiency, self multiplication and random neutron rate via the following relations;

$\mathrm{R} 1=$ Singles counting rate $* \mathrm{Tm}$

$\mathrm{R} 2=$ Doubles counting rate $* \mathrm{Tm}$

$\mathrm{R} 1=\varepsilon *\left[\mathrm{Tm} * \mathrm{Mult} * v_{\mathrm{S} 1} * \mathrm{Fs}+\mathrm{Tm} * \mathrm{Mult} * \mathrm{~S} \alpha\right]$.

$\mathrm{R} 2=(\varepsilon * * 2) *[\mathrm{Tm} * \mathrm{Mult} * * 2 *$ $\left\{v_{\mathrm{S} 2}+(\right.$ Mult- 1$\left.) * v_{\mathrm{S} 1} * v_{\mathrm{I} 2} /\left(\mathrm{v}_{\mathrm{II}}-1\right)\right\} * \mathrm{Fs}+$ $\mathrm{Tm} *$ Mult $* 2 *\left\{(\right.$ Mult -1$\left.\left.) * v_{{ }_{12}} /\left(v_{\mathrm{II}}-1\right)\right\} * \mathrm{~S} \alpha\right]$.

The parameters $\mathrm{R} 1$ and $\mathrm{R} 2$ are related to the experimental Feynman observables FEY $_{\text {CORR }}$ and FEY $Y_{\text {MEAN }}$ via

$\mathrm{Rl}=\mathrm{Tm} * \mathrm{FEY} \mathrm{MEAN} / \Omega$

$\mathrm{R} 2=\mathrm{Tm} *\left(\mathrm{FEY}_{\mathrm{CORR}} /(\Omega * \mathrm{WW} 2)\right.$

$\mathrm{WW} 2=1-\{1-\exp (-\lambda * \Omega)\} /\{\lambda * \Omega\}$

$\varepsilon=$ fission neutron detection efficiency

Mult = Self multiplication

Fs $=$ Fissions per second per gram

$S \alpha=$ Random neutron rate from alpha + neutron reactions

$\mathrm{Tm}=$ Measurement Time

$v_{\mathrm{S} 1}=$ Average number of neutrons emitted per spontaneous fission

$v_{I I}=$ Average number of neutrons emitted per induced fission

$v_{S 2}=$ Reduced second moment of the spontaneous fission neutron probability distribution $v_{12}=$ Reduced second moment of the induced fission neutron probability distribution $\lambda=$ The neutron detector decay constant for fission neutrons

$\Omega=$ The inspection time interval width in microseconds

$W W 2=$ The reduction in the number of neutrons observed due to the finite inspection interval $\Omega$ and the detector die-away time constant $\lambda$. 
For the active interrogation of small mass samples, we make the following assumptions.

1. The mass is distributed in small quantities such that the self multiplication is identically one, i.e. Mult $=1$;

2. The Feynman observables FEY MEAN $_{\text {and }}$ FEY CORR $_{\text {are due solely to thermal neutron induced }}$ fission of the fissile material. Any contributions due to random neutrons and spontaneous fission are measured in the region outside the time domain for thermal neutron induced fission, that is in the time region from 6000 to 10000 microseconds after the neutron generator neutron beam burst, and are subsequently subtracted from FEY $_{\text {MEAN }}$ and FEY $_{\text {CORR }}$ in the thermal neutron induced fission region.

3. The induced rate of fission varies with time in correspondence with the thermal neutron flux, that is the rate of induced fission decays exponentially with time after the neutron generator neutron beam burst. $F s \rightarrow F a s(t)=A * \exp (-0.693 * t / \Psi)$, where $\Psi$ is the thermal neutron half-life.

Under the above assumptions, we expand the above formalism to describe active measurements thus:

$$
\begin{gathered}
\begin{array}{c}
\mathrm{R} 1(\mathrm{t})=\varepsilon *\left[\mathrm{Tm} * \mathrm{Fs} * v_{\mathrm{S} 1}+\mathrm{Tm} * \mathrm{Fas}(\mathrm{t}) * v_{\mathrm{II}}\right. \\
+\mathrm{Tm} * \mathrm{~S} \alpha]
\end{array} \\
\mathrm{R} 2(\mathrm{t})=(\varepsilon * * 2) *\left[\mathrm{Tm} * \mathrm{Fs} * v_{\mathrm{S} 2}+\operatorname{Tm} * \mathrm{Fas}(\mathrm{t}) * v_{\mathrm{L} 2}\right]
\end{gathered}
$$

Subtracting out the spontaneous and random contributions to R1 and R2:

$$
\begin{aligned}
\mathrm{R} 1_{\text {ACTIVE }} & =\mathrm{R} 1(\mathrm{t})-\mathrm{R} 1_{\text {PASSIVE }} \\
& =\varepsilon *\left[\operatorname{Tm} * \mathrm{FaS}(\mathrm{t}) * v_{\mathrm{II}}\right] \\
\mathrm{R} 2_{\text {ACTIVE }} & =\mathrm{R} 2(\mathrm{t})-\mathrm{R} 2_{\text {PASSIVE }} \\
& =(\varepsilon * * 2) *\left[\operatorname{Tm} * \operatorname{Fas}(\mathrm{t}) * v_{\mathrm{I} 2}\right]
\end{aligned}
$$

Taking the ratio of $R 2_{A C T I V E}$ to $R 1_{A C T I V E}$ we obtain a simple linear dependence upon the absolute efficiency $\varepsilon$ and the ratio of the first and second induced moments:

$\mathrm{R} 2_{\mathrm{ACTIVE}} / \mathrm{R} 1_{\mathrm{ACTIVE}}=\varepsilon * v_{\mathrm{II}} / \mathrm{v}_{\mathrm{II}}$.

We can compare this result to the experimentally obtained N1 and N2, via:

$$
\begin{aligned}
& \mathrm{R}_{\mathrm{EXP}}=\mathrm{Tm} * \mathrm{FEY} \\
&=\mathrm{N} 1 / \Omega \\
& \mathrm{R} 2_{\mathrm{EXP}}=\mathrm{Tm} * \mathrm{FEY} / \Omega \\
&=\mathrm{N} 2 /(\Omega * \mathrm{WW} 2) .
\end{aligned}
$$

Taking the ratio $\mathrm{R} 2_{\mathrm{EXP}} / \mathrm{R} 1_{\mathrm{EXP}}$ we obtain

$$
\mathrm{R} 2_{\mathrm{EXP}} / \mathrm{R} 1_{\mathrm{EXP}}=\mathrm{N} 2 /(\mathrm{N} 1 * \mathrm{WW} 2) \text {. }
$$

From the Rossi Alpha time distribution measured for fission neutrons from a plutonium metal source we have extracted $\lambda$ to be $0.0245 /$ microsec for the shielded detectors of CTEN. Then for the analysis of the data with $\Omega$ of 100 microseconds, $\mathrm{WW} 2=0.627$, and

$\mathrm{R} 2_{\mathrm{EXP}} / \mathrm{R} 1_{\mathrm{EXP}}=\mathrm{N} 2 /(\mathrm{N} 1 * 0.627)$.

The average ratio of $\mathrm{N} 2 / \mathrm{N} 1$ formed by summing the values for $\mathrm{N} 1$ and $\mathrm{N} 2$ over the time region from 800 to 4000 microseconds after the neutron generator $14 \mathrm{MeV}$ neutron burst was observed to be $0.1414 \pm 0.0026$ for the 7 gram plutonium sample. From a similar measurement for the 9 gram ${ }^{235} \mathrm{U}$ sample the average ratio of $\mathrm{N} 2 / \mathrm{N} 1$ of $0.1119 \pm 0.0044$ was obtained.

From previous work with a well characterized neutron detector (Ref. 5) a 100 gm Pu metal sample has been observed to have a neutron source strength of 6828 neutrons/sec. This source produces a background corrected counting rate for the shielded ${ }^{3} \mathrm{He}$ detectors of the CTEN instruments of 681 hertz. An absolute detection efficiency of 0.0997 is obtained from the ratio of the counting rate to the neutron emission rate. In Table I are listed experimental results for the ratio of the second and first induced moments of the fission neutron emission probabilities for ${ }^{239} \mathrm{Pu}$ and ${ }^{235} \mathrm{U}$ obtained from this experiment using the expanded model of Hage and Cifarelli (Ref. 3) compared with the results of Boldeman and Hines (Ref. 6). The values for the ratio $v_{12} / v_{11}$ obtained from this simple expanded model applied to the experimental values of $\mathrm{N} 2 / \mathrm{N} 1$ from the present 
Table I. The experimental results for $v_{12} / v_{11}$ for the fission neutron emission probabilities for ${ }^{239} \mathrm{Pu}$ and ${ }^{235} \mathrm{U}$ obtained from this experiment compared with the results of (Ref.6).

\begin{tabular}{cccc}
\hline $\begin{array}{l}\text { Fissile } \\
\text { Material }\end{array}$ & $\mathrm{N} 2 / \mathrm{N} 1$ & $\begin{array}{r}v_{\mathrm{I} 2} / v_{\mathrm{I1}} \\
(\mathrm{exp})\end{array}$ & $\begin{array}{c}v_{\mathrm{I} 2} / v_{\mathrm{Il}} \\
\text { (Ref. 6) }\end{array}$ \\
\hline${ }^{239} \mathrm{Pu}$ & 0.1414 & 2.262 & 2.353 \\
& \pm .0026 & \pm .042 & \pm .017 \\
& & & \\
${ }^{235} \mathrm{U}$ & 0.1119 & 1.795 & 1.923 \\
& \pm .0044 & \pm .072 & \pm .008 \\
\hline
\end{tabular}

measurements are remarkably close to those obtained in Ref. 6.

\section{Determination of the transmission of fission neutrons through matrix systems.}

The 620 milligram sample of ${ }^{239} \mathrm{Pu}$ was inserted into a thin walled copper tube that could readily be inserted into one of the reentrant aluminum tubes present in the 55 gallon mock waste drums. The ${ }^{239} \mathrm{Pu}$ sample was then placed at three or four positions in each of three drums containing various amounts of polyethylene, and one drum containing graphite pieces as the matrix medium. The positions used were at radii of $0,12,20$ and $25 \mathrm{~cm}$. All were at $40 \mathrm{~cm}$ from the top of the tube, that is, near the middle of the drum height. The drums selected contained $9.5 \mathrm{~kg}$ of polyethylene shavings, $29.5 \mathrm{~kg}$ of polyethylene beads in vermiculite, $59.0 \mathrm{~kg}$ of polyethylene beads in vermiculite, and carbon block pieces. An active interrogation was performed at each position for 19992 neutron generator bursts. The drum was evenly rotated once during the interrogation. The data from the shielded detectors recorded with the PATRM was written to disk for off line analysis. A correlation window width of 100 microseconds was selected for the data analysis. Values for N1 and N2 were extracted from the Feynman distributions as described above, and average values for the ratio $\mathrm{N} 2 / \mathrm{N} 1$ were obtained. According to the extended Hage-Cifarelli model developed above, this ratio depends linearly upon the detection efficiency for the fission neutrons and the ratio $v_{12} / v_{11}$

$$
\mathrm{R} 2_{\mathrm{ACTIVE}} / \mathrm{R} 1_{\mathrm{ACTIVE}}=\varepsilon * v_{\mathrm{I}} / v_{\mathrm{II}} \text {. }
$$$$
\mathrm{N} 2 / \mathrm{N} 1=\varepsilon * v_{12} / v_{11} * \mathrm{WW} 2 .
$$

For the case in which the fissioning system is embedded within some matrix, such that only a fraction of the fission neutrons are transmitted through the matrix with sufficient energy to be detected by the shielded ${ }^{3} \mathrm{He}$ detectors, the efficiency above is replaced with

$\varepsilon \rightarrow \xi * \operatorname{TRAN}$,

where TRAN is the transmission value, that is the fraction of neutrons transmitted through the matrix, and $\varepsilon$ is the absolute fission neutron detection efficiency for the CTEN system with an empty chamber. Thus the transmission and measured values of $\mathrm{N} 2 / \mathrm{N} 1$ should be related via

$\mathrm{N} 2 / \mathrm{N} 1=\xi * \operatorname{TRAN} * v_{12} / v_{11} * \mathrm{WW} 2$.

In order to examine the above relationship we determined the transmission of fission neutrons through the matrix into the shielded ${ }^{3} \mathrm{He}$ detectors at each of the three, or four, locations within each drum for which we measured the N2/ N1 ratio. These transmissions were obtained by measuring the fission neutron counting rate from the 100 gram plutonium cylinder at each position, and making the appropriate correction for neutrons from cosmic radiation and other background sources. These rates were then divided by the background corrected counting rate for this source located in the middle of the empty CTEN chamber. The results for N2/ N1 and the transmission obtained for the fourteen measurements within the four types of matrix, as well as for the 7 gram dispersed Pu oxide sample located in the empty CTEN chamber, representing $100 \%$ transmission, are shown graphically in Fig. 2. A linear relationship is assumed and the straight line in the figure has a slope of 0.1414 with an intercept of zero. These results imply that for moderating matrix materials of either hydrogen or carbon, the ratio N2/ N1 can be used to determine the fraction of fission neutrons that are transmitted through the matrix into the ${ }^{3} \mathrm{He}$ detectors. For these cases, it is then possible to cleanly separate the effects of the matrix into two processes, one which alters the thermal neutron interrogating flux, and one which alters the fission neutron transmission to the detectors. The study of the process which alters the thermal neutron interrogation flux is now undergoing extensive investigations. Preliminary 


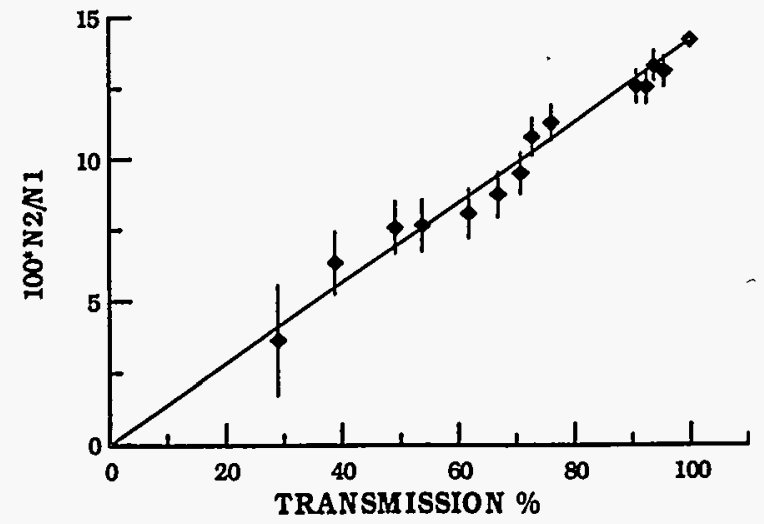

Fig. 2. The variation of the ratio $100 * \mathrm{~N} 2 / \mathrm{N} 1$ with the transmission for the polyethylene and carbon matrix materials.

results indicate that for the case of homogenous matrix materials which contain hydrogen and no other significant thermal neutron absorbing material, corrections for the thermal flux variation as a function of location within the drum are possible, and a substantially improved mass determination can be achieved.

\section{References}

1. G. J. Arnone, G. S. Brunson, and K. L. Coop, 1992 IEEE Nuclear Science Symposium, Orlando, Florida.

2 .R. P. Feynman, F. DeHoffmann, and R. Serber, Journal of Nuclear Energy, 3, (1956) 64.

3. D. M. Cifareli and W. Hage, Nucl. Instr. and Meth. A251, (1986) 550.

\section{Conclusions}

We have observed correlations in the neutron signal produced by small samples of ${ }^{239} \mathrm{Pu}$ and ${ }^{235} \mathrm{U}$ under active interrogation with thermal neutrons in the CTEN instrument. The values for the ratio $v_{12} v_{11}$ deduced from these measurements using a modified version of the model of Hage and Cifareli are in good agreement with previous published results. The number of correlated neutrons provides a second parameter for use in conjunction with the total number of neutrons observed in an active assay of small samples of fissionable material. We have observed that the ratio N2/N1 can be used to determine the fraction of fission neutrons that are transmitted through the matrix into the ${ }^{3} \mathrm{He}$ detectors. For the case is which sufficient ${ }^{240} \mathrm{Pu}$ is present to allow a passive assay, the transmission result obtained in the active assay can be used in the multiplicity analysis of the passive data. This independent determination of the "efficiency" reduces the number of parameters needed to be extracted by the multiplicity analysis and substantially improves the accuracy of the assay.

4. A. A. Robba, E. J. Dowdy, and H. F. Atwater, Nucl. Instr. and Meth. 215, (1983) 473.

5. G. S. Brunson and G. J. Arnone, LA-11701 MS, November 1989

6. J. W. Boldeman and M. G. Hines, Nuclear Science and Engineering, 91, (1985) 114. 